\title{
A New Method for SPECT Quantification of Targeted Radiotracers Uptake in the Myocardium
}

\author{
Shimin Li, Lawrence W. Dobrucki, Albert J. Sinusas, and Yi-Hwa Liu \\ Section of cardiovascular Medicine, Department of Internal Medicine, \\ Yale University School of Medicine, New Haven, CT, USA \\ Shimin.1i@yale.edu
}

\begin{abstract}
We developed a new method for absolute quantification of targeted radiotracers uptake in the myocardium using hybrid SPECT/CT and an external reference point source. A segmentation algorithm based on the level set was developed to determine the endocardial edges from $\mathrm{CT}$, which were subsequently applied to the physically co-registered SPECT. A 3-D Gaussian fitting method was applied for quantification of the external point source. The total targeted radiotracer activity in the myocardium was normalized to that in the point source to calculate the absolute uptake of targeted radiotracer in the myocardium. Preliminary validation was performed in rats with ischemia-induced angiogenesis. The quantified in vivo radiotracer uptake was compared to the postmortem tissue radioactive well-counting of the myocardium. Our methods worked well for identification of the endocardial edges. Quantification of the focal uptake was consistent with the well-counting data. Our methods may have the potential of providing precise absolute quantification of targeted radiotracer uptake in the myocardium.
\end{abstract}

\section{Introduction}

Recently substantial research was directed toward the developments of targeted molecular imaging agents [1] for the cardiovascular system and radiotracer based imaging techniques for noninvasive visualization of the molecular processes in the myocardium. Radio-labeled agents targeted at the molecular processes often result in focal "hotspot" images, which are distinctly different from the perfusion images conventionally acquired in nuclear cardiology. Because the changes in the myocardial uptake of these targeted radiotracers are generally small, precisely tracking these subtle changes requires a sophisticated quantitative method to assess the absolute hotspot uptake in the myocardium. However, quantification of myocardial uptake of targeted radiotracers has not been extensively investigated previously in part due to the background activity in the cardiac images. Quantification of hotspot focal uptake in the myocardium from single photon emission computerized tomography (SPECT) presents another challenge due to the effects of image resolution, background activity $[2,3]$, object size, shape and voxel size in SPECT images [4]. Other confounding factors such as photon attenuation and the partial volume effect may also cause errors in quantification of absolute hotspot tracer uptake [5] in the myocardium. On the other hand, it is difficult to obtain anatomical information from focal hotspot SPECT 
images and thus reference images such as ${ }^{201} \mathrm{Tl}$ or $\mathrm{CT}$ images are needed to identify the position and orientation of the heart. Currently, quantitative approaches are not available for serial evaluation of changes in the uptake of these targeted radiotracers. Previous studies in quantification of targeted cardiac images are mostly derived only from visual analysis. Recently, we developed a stochastic approach [6] for quantification of focal uptake from cardiac SPECT/CT images using a ${ }^{99 \mathrm{~m}} \mathrm{Tc} /{ }^{201} \mathrm{Tl}$ dual-isotope SPECT imaging protocol in which ${ }^{201} \mathrm{Tl}$ SPECT was used as the reference perfusion image. However, quantification of the targeted radiotracer uptake using two isotopes may require assumptions of the reciprocal relation between the ${ }^{99 \mathrm{~m}} \mathrm{Tc}$ and ${ }^{201} \mathrm{Tl}$ images which may not be true due to the cross-talk between the two tracers on board. Using $\mathrm{CT}$ image to replace ${ }^{201} \mathrm{Tl}$ image as the reference image may potentially address this issue.

In this paper, we introduce a new level-set-based SPECT/CT segmentation and quantification approach for absolute quantification of myocardial uptake of radiotracers targeted at the molecular processes such as angiogenesis. Absolute myocardial uptake is quantified from SPECT images with the support of co-registered SPECT/CT images in identification of the left ventricular (LV) myocardium.

\section{Methods}

\subsection{The Level Set Approach for Image Segmentation}

The level set method, developed previously by Osher and Sethian [7, 8], is a numerical approach for tracking the evolution of the surfaces. Let $\Gamma(t)$ denote a timedependent closed (n-1)-dimensional hyper-surface which evolves in its normal direction. Instead of maneuvering the hyper-surface directly, the desired hyper-surface is embedded in the zero level set of a higher $n$-dimensional function $\phi(x, t)$, which can be described as:

$$
\Gamma(t)=\{x \mid \phi(x, t)=0\},
$$

The value of $\phi$ at point $x$ is defined by:

$$
\phi(x, t)= \pm d,
$$

where $d$ is the shortest distance from $x$ to the desired hyper-surface $\Gamma(t)$. The sign is determined by either the point $x$ lying outside $(+\mathrm{d})$ or inside (-d) the hyper-surface $\Gamma(t)$. The level-set function is evolved under the control of the differential equation:

$$
\frac{\partial \phi(x, t)}{\partial \tau}+F|\nabla \phi(x, t)|=0,
$$

where $|\nabla \phi|$ is the norm of the gradient of the level set function $\phi$. The speed term $F$ in Equation 3 to be defined in the next section depends on the feature of the image segmented and the intrinsic geometrical property of the surface (eg. curvature of the surface). Given $\phi(x, t)=0$, the evolving surface can be obtained by extracting the zero level-set as expressed in Equation 1. For details of the level set methods, the reader can refer to [9]. 


\subsection{The Cavity Separation}

In vivo cardiac CT images have very low contrast and ambiguous boundaries due to the cardiac motion. Segmentation of the myocardium from the in vivo CT images is challenging. Instead of segmenting the myocardium, we segment the cavity from the in vivo CT images because of the complex shape of the myocardium. We also rescale the $\mathrm{CT}$ images to enhance the intensity in the $\mathrm{LV}$ regions. Let $I_{C}$ denote the rescaled CT image. The speed term used in Equation 3 can be formulated as [9]:

$$
F=(\alpha-\beta \cdot \kappa) \cdot g_{I_{C}}-\gamma \frac{\nabla g_{I_{C}} \cdot \nabla \varphi}{|\nabla \varphi|},
$$

where $g_{I_{c}}=\frac{1}{1+\left|\nabla\left(G_{\sigma} * I_{C}\right)\right|}$ and $\kappa=\nabla \cdot \frac{\nabla \phi}{|\nabla \phi|}$. The expression $G_{\sigma}{ }^{*} I_{C}$ denotes the rescaled CT images convolved with a Gaussian smoothing filter of which the characteristic width is denoted as $\sigma$. We use the Gaussian filter to reduce the noise of the edges and facilitate the edge detection for the anatomical structures of the LV. $\kappa$ is a curvature-based geometric smoothing term. The last term $\frac{\nabla g_{I_{C} \cdot} \cdot \nabla \phi}{|\nabla \phi|}$ in Equation 4 is used to direct the curve to the boundaries of the LV cavity. The scalars $\alpha, \beta$ and $\gamma$ are the parameters used to weigh the relative influence of each of the terms on the movement of the edges.

Proper initialization is critical for the level set algorithm. A seed region with one or more pixels needs to be manually introduced into the cavity volume. Ultimately, the cavity volume is updated iteratively with the speed term $F$ until it reaches the endocardial edges.

\subsection{Point Source Extraction}

In order to calculate the total counts of the external reference point source, the volume of the reference point source needs to be extracted from the SPECT images. A 3-D Gaussian fitting is incorporated into the point source extraction. Total counts of the external point source fitted as a 3-D elliptical Gaussian shape are integrated from the center of the volume to 2 standard deviation (SD) outward in the 3-D space to acquire $97.5 \%$ of the counts in the elliptical shape. The 3-D Gaussian function of the point source can be formulated as

$$
P(x, y, z)=A \cdot \exp ^{-\frac{1}{2}\left(X-X_{n}\right)^{T} M\left(X-X_{n}\right)},
$$

Where $X=(x, y, z)^{T}$ denote the spatial coordinates, $X_{n}=\left(x_{n}, y_{n}, z_{n}\right)^{T}$ denote the spatial coordinates of the center of the point source, $A$ is the height of the Gaussian shape, and $M=\left(\begin{array}{ccc}\frac{1}{\sigma_{x}^{2}} & 0 & 0 \\ 0 & \frac{1}{\sigma_{y}^{2}} & 0 \\ 0 & 0 & \frac{1}{\sigma_{z}^{2}}\end{array}\right)$. The variance of the Gaussian in different directions 
( $\left.\sigma_{x}, \sigma_{y}, \sigma_{z}\right)$ are not necessarily the same. The parameters of the point source distribution can be estimated by a gradient-expansion algorithm [10] to compute a non-linear least squares with the seven parameters shown above.

To locate the point source in the SPECT images, a seed is manually introduced into the region of the point source. The initial center of the point source can be defined by the position of maximal value pixel in the finite region around the seed. After the parameter estimation, the count of the point source can be collected from the 3-D elliptical volume with the estimated standard deviations.

\subsection{Quantification of Targeted Radiotracer Uptake from SPECT Images}

The signed distance map as described in Section 2.1 can be generated with incorporation of the LV cavity volume obtained from the CT images. In the signed distance map, pixel values represent the shortest signed distance of the points from the contour. Consequently, the myocardial volume is obtained by integrating all the pixels within an assumed myocardial thickness. The myocardium determined by CT can be applied to the targeted radiotracer SPECT images (see Fig. 1(c)) thanks to the coregistration of the SPECT and CT images.

The point source filled with ${ }^{99 \mathrm{~m}} \mathrm{Tc}$ was used as a known reference to quantify absolute uptake of the targeted radiotracers in the LV myocardium. The boundary between the left ventricular and left atrial myocardium was determined manually and was located approximately one half of the whole LV length as evidenced by the CT images. Targeted radiotracer uptake in the LV myocardium was normalized to the total counts of the external point source of which the volume was determined by the method described in Section 2.3. Relative radiotracer uptake was calculated as a summation of pixels values in the segmented LV myocardium volume. The resulted relative radiotracer uptake was in turn weighted by the known dose of the external point source to obtain absolute radiotracer uptake. The total absolute radiotracer uptake is calculated by

$$
\text { Targeted radiotracer uptake }=\frac{\iint \zeta(v) d v}{\iint \theta(u) d u} \cdot c,
$$

where $\zeta(v)$ denotes the targeted activity in the LV myocardium volume $v, \theta(u)$ denotes the targeted activity in point source volume $u$, and $c$ represents the known radioactivity $(\mu \mathrm{Ci})$ in the point source.

\section{Rat Experiments}

\subsection{Surgical and Imaging Preparation}

Rats weighting 200-250 gm were used in our preliminary validation for the methods. The rats were anesthetized with isoflurane and the hearts was exposed via a limited left anterolateral thoracotomy. The left anterior descending coronary artery was ligated at $7 \mathrm{~mm}$ distal to the origin. The coronary occlusion was subsequently released after 45 minutes resulting in non-transmural infarction and the chest was closed in layers. One week following myocardial infarction, the rats were anesthetized with 
isoflurane and positioned in a hybrid microSPECT/CT imaging system (XSPECT Gamma Medica, Northridge, CA) for microCT and microSPECT imaging. A point source filled with $32 \mu \mathrm{Ci}{ }^{99 \mathrm{~m}} \mathrm{Tc}$ was placed under the imaging table and near the rat. A ${ }^{99} \mathrm{~m}$ Tc-labeled radiotracer $(4 \sim 5 \mathrm{mCi})$ targeted at the $\alpha \mathrm{v} \beta 3$ integrin $(\mathrm{NC100692}, \mathrm{GE}$ Healthcare), a marker of angiogenesis, was injected into the rats. The rats were also injected with an intravascular vascular contrast agent (Fenestra VC, Alerion Biomedical, San Diego, CA) to visually separate the myocardium from LV cavity.

\subsection{Image Acquisitions and Reconstructions}

Non-gated CT projections were acquired for 15 minutes. A total of 512 projections were acquired. The $\mathrm{CT}$ images were reconstructed using the filtered back-projection algorithm. The matrix size of CT images was $512 \times 512 \times 512$ voxels. The voxel size of CT reconstruction was $0.1557 \mathrm{~mm}$ cubic.

After CT imaging, SPECT images were acquired using the same dual-head microSPECT/CT camera with pinholes collimators ( $1 \mathrm{~mm}$ aperture) for 35 minutes, with a $20 \%$ energy window symmetrically centered at $140 \mathrm{keV}$ photopeak of ${ }^{99 \mathrm{~m}} \mathrm{Tc}$. Eighty-two projections were acquired for each head in a $180^{\circ}$ circular orbit. The SPECT images were also reconstructed using the filtered back-projection algorithm. The matrix size of SPECT images was $82 \times 82 \times 82$ voxels, with voxel size of 1.524 $\mathrm{mm} \times 1.524 \mathrm{~mm} \times 1.524 \mathrm{~mm}$. CT and SPECT images were fused to obtain co-registered SPECT/CT images. All fused images had an image matrix size of $256 \times 256 \times 256$ voxels, with voxel size of $0.3114 \mathrm{~mm} \times 0.3114 \mathrm{~mm} \times 0.3114 \mathrm{~mm}$.

\subsection{CT Image Segmentation and SPECT Image Quantification}

The level set image segmentation method was applied to the CT images to determine the endocardial edges and the resulted edges were subsequently applied to the SPECT images to calculate the focal radiotracer uptake in the LV myocardium as described in Section 2.

\subsection{Tissue Well-Counting}

Rats were sacrificed after SPECT/CT imaging. The heart was extracted from the chest and was sliced for tissue well-counting measurements of the radioactivity in the LV myocardium. The well-counting data was corrected for decay based on the time interval between the SPECT imaging and well-counting.

\section{Results}

Fig.1(a) illustrates the in vivo CT images for one rat. The cavity of the left ventricle determined using the level set method is shown in Fig. 1(b) in red and the myocardium between the endocardial and epicardial edges is shown in green. The thickness of the myocardium shown in Fig.1(b) was seven pixels $(\sim 2.2 \mathrm{~mm})$. The LV edges were subsequently applied to the targeted SPECT images as shown in Fig. 1(c) to integrate the total photon counts in the targeted regions in the LV myocardium. The 


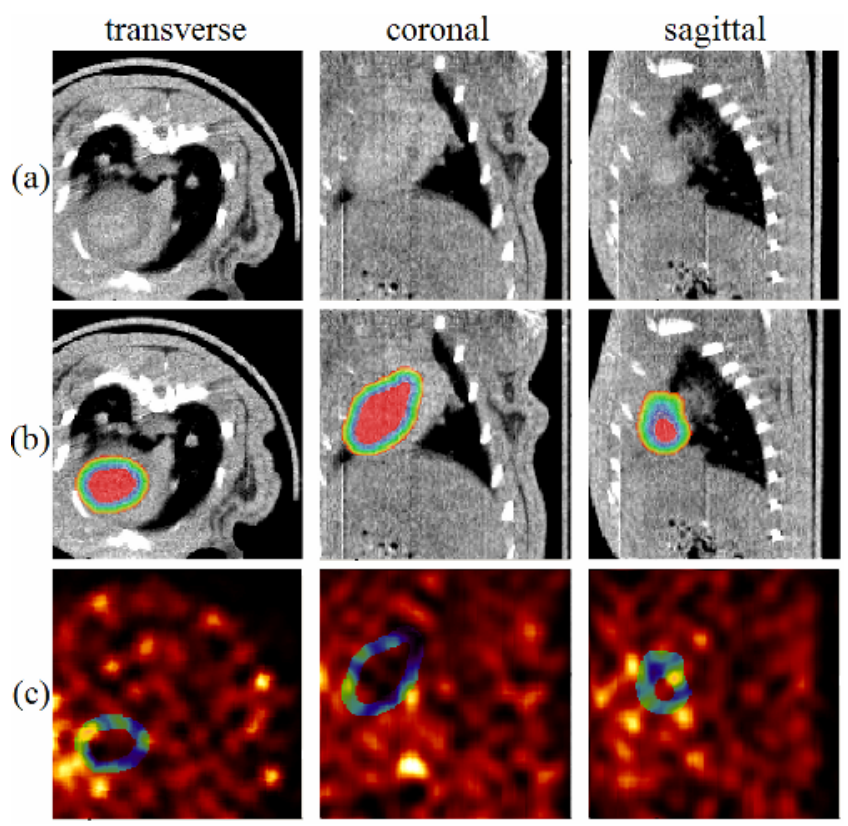

Fig. 1. (a) In vivo CT images, (b) In vivo CT images with LV cavity (in red) and myocardium (in green) superimposed, (c) In vivo SPECT images superimposed with the LV myocardial edges from (b). The myocardial thickness is 7 pixels width in this illustration.

integrated tracer counts were normalized to that in the point source to calculate the absolute radiotracer dose in the LV myocardium as described in Section 2.4. Point source dose $(\mu \mathrm{Ci})$ was calculated using the method described in Section 2.3. Because only one point source was used in our experiments, the absolute dose in the point source was decay corrected for each rat.

Table 1 shows the well-counting dose and SPECT quantified doses with 7 different wall thicknesses assumed in our segmentation method. The ratio shown in Table 1 was calculated as the SPECT dose divided by the true well-counting dose. The wellcounting data was also corrected in the same way based on the time interval between the SPECT imaging and well-counting. As seen in Table 1, the ratio is linearly increased as a function of the assumed LV myocardium thickness. The positive linearity is expected because a thicker LV thickness assumed should reflect higher integrated counts from the myocardium. Notice that the SPECT quantified dose using sevenpixel thickness $(\sim 2.2 \mathrm{~mm})$ resulted in the smallest error as compared to the other thicknesses used. The true thickness of rat myocardium is approximately $1 \sim 1.5 \mathrm{~mm}$. By taking into account the intrinsic pinhole SPECT resolution $(\sim 1 \mathrm{~mm})$ and low-pass filtering effect on SPECT images, the myocardial thickness assumed in SPECT images needs to be at least 2 times image resolution to compensate for the partial volume effect. This may explain that the assumed seven-pixel thickness resulted in a better estimation of the uptake of the targeted radiotracer. 
Table 1. Comparisons between the SPECT quantified radiotracer dose and true well-counting dose in 7 different thicknesses assumed for the left ventricular myocardium of rat

\begin{tabular}{cccccccc}
\hline Rat \# & $\begin{array}{c}\text { Well } \\
\text { counting } \\
\text { dose } \\
(\mu \mathrm{Ci})\end{array}$ & $\begin{array}{c}\mathrm{D}=4 \text { pixels } \\
\text { SPECT } \\
\text { dose } \\
(\mu \mathrm{Ci})\end{array}$ & Ratio & $\begin{array}{c}\mathrm{D}=5 \text { pixels } \\
\text { SPECT } \\
\text { dose } \\
(\mu \mathrm{Ci})\end{array}$ & Ratio & $\begin{array}{c}\mathrm{D}=6 \text { pixels } \\
\text { SPECT } \\
\text { dose } \\
(\mu \mathrm{Ci})\end{array}$ & Ratio \\
\hline 1 & 1.93 & 0.76 & 0.39 & 1.08 & 0.56 & 1.56 & 0.81 \\
2 & 1.56 & 0.75 & 0.48 & 1.09 & 0.70 & 1.53 & 0.98 \\
\hline $\mathrm{D}=7$ pixels & $\begin{array}{c}\mathrm{D}=8 \text { pixels } \\
\text { SPECT } \\
\text { dose } \\
(\mu \mathrm{Ci})\end{array}$ & Ratio & $\begin{array}{c}\mathrm{D}=9 \text { pixels } \\
\text { SPECT } \\
\text { dose }\end{array}$ & Ratio & $\begin{array}{c}\mathrm{D}=10 \text { pixels } \\
(\mu \mathrm{C} i)\end{array}$ \\
$\begin{array}{c}\text { dose } \\
\text { dose }(\mu \mathrm{Ci})\end{array}$ & Ratio & Ratio \\
\hline 1.91 & 0.99 & 2.56 & 1.33 & 3.17 & 1.65 & 3.93 & 2.03 \\
1.94 & 1.24 & 2.52 & 1.62 & 3.11 & 1.99 & 3.82 & 2.45 \\
\hline
\end{tabular}

\section{Discussion and Conclusion}

We have derived an image segmentation method using the level set scheme to identify the LV cavity in CT images and developed a SPECT quantification approach for assessment of absolute targeted radiotracer uptake in the LV myocardium. A hybrid microSPECT/CT imaging system was used for imaging. The methods were evaluated using an in vivo rat model and a radiotracer targeted at angiogenesis. The validity of the results was demonstrated in 2 rats with 7 different myocardium thicknesses assumed in our methods. The linearity of SPECT quantified radiotracer uptake with respect to the thickness confirmed that the results of absolute radiotracer uptake quantification in our rat data were quite promising. Using the 7-pixel wall thickness in consideration of image resolution and the partial volume effect, the resulted SPECT quantified dose was comparable to the true dose obtained from the wellcounting data.

While the preliminary results presented herein are encouraging, we did not apply photon attenuation and scatter corrections to the SPECT images in this animal validation. We believe that the estimation of absolute quantification of targeted radiotracer uptake in the LV myocardium can be further improved when these corrections are incorporated. Also, the level sets used in the myocardium segmentation may be affected by the complicated parameter settings and the initial placement of seed region in the cavity. Thus, further validation of our methods in adjusting those settings is warranted.

\section{Acknowledgments}

This work was supported in part by the National Institutes of Health (NIH) under Grants R21-EB001774 and R01-HL65662. The authors would like to thank Xiao-Yu Hu, MD 
and Patti Cavaliere for their assistance in the animal preparation and surgery. The authors also acknowledge that the NC100692 was provided by GE Healthcare.

\section{References}

1. Okada R.D., Johnson G., et al.: 99mTc-HL91: "hot spot" detection of ischemic myocardium in vivo by gamma camera imaging. Circulation, 97: 2557-66, 1998.

2. Kessler R.M., et al.: Analysis of emission tomographic scan data: limitations imposed by resolution and background. J Comput Assit Tomogr, 8:514:522, 1984.

3. Kojima A., Matsumoto M., et al.: Effect of spatial resolution on SPECT quantification values. J Nucl Med, 30:508-514, 1989.

4. King M.A., Long D.T., Brill A.B.: SPECT volume quantitation: influence of spatial resolution, source size and shape, and voxel size. Med. Phys, 18:1016-1024, 1991.

5. Da Silva A.J., Tang H.R., Wong K.H., et al.: Absolute in vivo quantitation of myocardial activity. IEEE Trans. Nucl Sci, 47:1093-1098, 2000.

6. Liu Y.H., Li S., Bourke B., Weyman C., Sinusas A.J.: A stochastic approach for quantification of hotspot focal uptake from cardiac SPECT imaging: a canine validation. J Nucl Cardiol, 12:S73,2005.

7. Osher S., Sethian J.: Fronts propagating with curvature dependent speed:algorithms based on the Hamilton-Jacobi formulation. J. Comp. Phys, 79:12-49, 1988.

8. Malladi R., Sethian J., Vemuri B.: Shape modeling with front propagation: A level set approach. IEEE Trans. PAMI, 17(2):158-175, 1995.

9. Caselles V., Kimmel R., Sapiro G.: Geodesic Active Contours. Int'l J. Comp. Vision, 22(1):61-79, 1997.

10. Marquardt D W.: An Algorithm for Least-Squares Estimation of Nonlinear Parameters. J. Soc. Indust. Appl. Math. 11:431-41, 1963. 